\title{
Essence of Human Intelligence
}

\section{Matthew N. O. Sadiku1*, Tolulope J. Ashaolu2, and Sarhan M. Musa1}

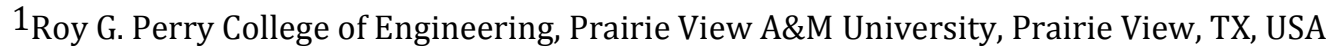 \\ ${ }^{2}$ College of Food Science, Southwest University, Tiansheng Road Beibei District, Chongqing, 400715, P.R. China \\ E-mail: sadiku@ieee.org; ashaolut@gmail.com; smmusa@pvamu.edu \\ *Corresponding author details: Professor Matthew N. O. Sadiku, sadiku@ieee.org
}

\begin{abstract}
Intelligence is a general mental ability for reasoning, problem solving, and learning. It is the most prominent quality that makes humans unique. Traditionally, intelligence is viewed as a fixed amount of cognitive capacity that people are born with and the capacity cannot be changed. Gardner's Multiple Intelligence Theory posits that individuals have different amounts, abilities, and unique combinations of intelligences that can be changed and improved upon throughout their lifetime. This paper presents an introduction to what human intelligence means, how it is developed or tested, and what role it plays in our life.
\end{abstract}

Keywords: intelligence; human intelligence; artificial intelligence; intelligence tests

\section{INTRODUCTION}

Intelligence is regarded as an individual's assessment of success in life by the individual's own standards. Success is achieved by using combinations of analytical, creative, and practical intelligence. Through intelligence, humans can do many things such as learning, reasoning, applying logic, recognizing patterns, solving problems, making decision, retaining information, managing emotions, and using language to communicate. Intelligence has historically been conceptualized as a more or less fixed trait. Some believe that you do not have much control over your intelligence. It was genetically determined at birth. Efforts to raise the intelligence of children (through programs like Head Start) are always abortive.

Figure 1 shows how psychologies define intelligence [1]. Intelligence is highly correlated with successful training and performance outcomes. Individuals with relatively less intelligence often work harder in order to achieve goals and compensate for their lack of intelligence. Such individuals will avoid challenges. They are more likely to divorce, have a child out of marriage, be incarcerated, and need long-term welfare support. On the other hand, persons with high intelligence are associated with more years of education, higher status jobs, and higher income [2].

\section{WHAT IS HUMAN INTELLIGENCE?}

Human intelligence is the mental capacities to learn, understand, and reason, including the capacities to comprehend ideas, plan, solve problems, and use language to communicate. It is the mental quality that consists of the abilities to learn from experience, adapt to new situations, understand abstract concepts, and use knowledge to adapt to the environment. It integrates cognitive functions such as perception, attention, memory, language, and planning. Figure 2 illustrated the three component of human intelligence [3].

Recent research efforts combining psychology, biology, neuroscience, anthropology, archaeology, and cognitive science are leading to an increasingly sophisticated understanding of how human intelligence evolved [4].
Since intelligence appears to depend on functions supported by different brain areas, it has been suggested that genetic engineering could be used to enhance intelligence. The mind may be regarded as a set of information-processing procedures (cognitive programs) that are embodied in the neural circuitry of the brain.

Sex, age, and culture play a role in shaping intelligence. Some psychologists have shown that human intelligence is unique to the culture. Human intelligence carries different meanings in different cultures around the world. In many Eastern cultures, Chinese, and African cultures, intelligence is shown through a social lens and exemplified through social responsibilities. Confucius' contributions to Chinese philosophical tradition are widely acknowledged in the West. Western conceptions dominate the study of intelligence around the globe.

\section{ASSESMENT OF HUMAN INTELLIGENCE}

Human intelligence is commonly assessed by IQ scores that are determined by IQ tests. An intelligence quotient (IQ) is a total score derived from a set of standardized tests to assess human intelligence. In the United States, IQ scores are important for school, employment, job performance, and military service as_an individual's IQ is incorporated into decision making. There are organizations which limit their membership to people who high IQ scores. The mental tests are based on a model that portrays intelligence as a composite of abilities and can be quantified. IQ was originally computed as the ratio of mental age to chronological age, multiplied by 100 . People differ along mental continua. Such individual differences in intelligence are usually measured using psychometric tests. Intelligence test scores and intelligence differences in the population both roughly follow a normal distribution. IQ tests as regarded as highly reliable, meaning that a reliable test produces similar scores upon repetition [5].

Intelligence tests are often used in educational, business, and military settings because of their efficacy in predicting behavior. There are also psychometric standardized tests that are not intended to measure intelligence itself but scholastic aptitude. In the United States examples include 
the SSAT, the SAT, the ACT, the GRE, the MCAT, the LSAT, and the GMAT, which produce intelligence scores [2].

\section{INCREASING YOUR INTELLIGENCE}

If you adopt the following five principles, you will be performing at your peak ability [6].

\section{Seek Novelty:}

Geniuses like Einstein are constantly seeking out novel activities, learning a new domain. People who seek novelty and rate high on openness are constantly seeking new information, new things to learn, new experiences, and new activities to engage and expand cognitive horizon.

\section{Challenge Yourself:}

Individual brain training games don't make you smarter. Once you master one of those cognitive activities in the brain-training game, you need to move on to the next challenging activity. The brain uses more energy during training times. To keep your brain making new connections and keeping them active, you need to keep moving on to another challenging activity as soon as you reach the point of mastery of the present activity.

\section{Think Creatively:}

Creative thinking involves recruitment from both halves of your brain, not just the right. It may involve making remote associations between ideas and generating original, novel ideas that are also appropriate to the activity you are doing.

\section{Do Things the Hard Way:}

Doing things efficiently is not doing your brain any favors. Technology does a lot to make things in life easier, faster, more efficient, but sometimes our cognitive skills can suffer as a result of these shortcuts. Your brain needs exercise. If you stop using your problem-solving skills, your spatial skills, your logical skills, your cognitive skills, your brain never improves. There are times when it is better to say no to shortcuts and use your brain, as long as you can afford the luxury of time and energy. Taking the stairs instead of the elevator a few times a week is highly recommended.

\section{Networking}

By exposing yourself to new people, ideas, and environments, you are opening yourself up to new opportunities for cognitive growth. You can do this through social media such as Facebook or Twitter, or in face-to-face interactions. Learning is all about exposing yourself to new things and seeing things from a new perspective. The greatest thing about networking is that everyone benefits.

ARTIFICAL INTELLENCE AND HUMAN INTELLIGENCE What distinguishes human intelligence from nonhuman/artificial intelligence?

Artificial intelligence (AI) describes intelligence demonstrated by machines as opposed to the intelligence possessed by humans. It is ability to mimic human behavior. It is the science of making smarter and intelligent machines that can think and act like humans. Such intelligent machines can learn about the world that surrounds them and take actions. Human intelligence shapes the emergence and adoption of artificial intelligence. The one area where the power of human intelligence really shines is innovation. The rise of $\mathrm{AI}$ is raising the question on tasks that only humans can do. It prompted much concern about the fate of human jobs in the wake of AI. This has sparked an inevitable debate between $\mathrm{AI}$ and human intelligence. Artificial intelligence and human intelligence can be compared as follows [7, 8]:

- Field: AI belongs to computer science, while human intelligence (HI) is in psychology.

- Speed of Execution: If one doctor can make a diagnosis in 10 minutes, AI system can make a million diagnoses for the same time.

- Less Biased: Humans can be biased but AI system is not biased in decision making process.

- Operational Ability: Humans need a break, AI system works tirelessly.

- Accuracy: An AI system is more accurate and precise than humans.

- Memory Usage: Humans use content memory and thinking, while robots are use built-in instructions.

- Mode of Creation: Human intelligence is bigger because it is created by God and artificial intelligence is artificial and designed by humans.

- Learning Process: Human intelligence responses to millions of functions overall in their lives, whereas AI is developed for specific tasks only.

- Decision Making: AI systems are clueless in the understanding of "cause" and "effect". They cannot make decisions like humans since they lack "common sense."

Figure 3 depicts the comparison of artificial intelligence and human intelligence [9]. Some experts have predicted that with time, AI will exceed human intelligence. Machines are still away from what human brains are capable of doing. They will augment human tasks, but not replace them. It will not be possible for such machines to completely replace the human resource. The system that integrates both humans and machines is variably known as hybrid intelligence, extended intelligence or ambient intelligence [10]. It is shown in Figure 4 [11]. The need for such systems is already acknowledged in critical domains such as medicine and driving.

\section{APPLICATIONS}

Intelligence is important in different fields such as in education, military, and scientific inquiry.

- Education:

Education practitioners and school administrators can benefit from learning more about human intelligence. Educators need that which enables competent action. It is their desire to equip students with what they will need to navigate successfully the world. They consider intelligence as the ability to reason and adapt. Any excellence in academic performance will be related to intelligence in some way. Standardized tests have in one form or another have been used to measure intelligence. Intelligence tests are used predominantly for children who are considered intelligent. Children are full time scientists, researching the world as they play [12].

- Military Intelligence:

Human intelligence (HUMINT) is part of military intelligence, which applies to the full range of military operations. The discipline elicits intelligence through interactions with human sources. Human intelligence plays a crucial role in US national and foreign security policy and in protecting US interests. The US Army should be the first within the US Government to understand that decision support information (intelligence regardless of classification) is the key to creating and stabilizing the earth. We are unwisely spending $\$ 75$ billion a year on global secret technical collection efforts, while spending relatively nothing on processing, or interagency sharing of data, or on decision support. Domestic intelligence operations must evolve to meet present and future threats $[13,14]$. 
- Human Genetics:

Humans have brains with significantly increased size and complexity compared to their ape counterparts. Various approaches in molecular biology have been used to search for the human-specific genes and mutations therein that were related to the human intelligence. Understanding the evolution of human intelligence is an important undertaking in the science of human genetics. Genetic changes affecting intelligence in hominid evolution have remained elusive [15].

\section{- Intelligent Buildings:}

Human intelligence is gaining popularity within the "green building" and "intelligent building" discussion. Advocates of intelligent buildings consider greater automation as being necessary to provide high environmental performance. Intelligence comes in the operational efficiency of buildings and their effectiveness to provide functionality and occupant comfort. The type and extent of "automated" and "human" intelligence in buildings can affect issues ranging from energy and operational efficiency to inhabitant satisfaction, productivity, security, and privacy. There is no single or correct approach to achieving intelligent buildings [16].

Other applications of human intelligence include collective intelligence,_environment, and religiosity.

\section{CHALLEGES}

Some have raised the ethical question: Why do we need to design thinking, intelligent machines when humans are better equipped to think faster than machines? Conscious efforts to influence intelligence also raise ethical issues. Computer modeling is yet to resolve some major problems in understanding the nature of intelligence Standardized intelligence tests have incurred some criticism. There are critics who argue that to base intelligence on IQ test scores alone is to ignore many important aspects of mental ability. Some argue that intelligence tests should not be used in identifying giftedness. Many programs and important college decisions are based on the result of a single-shot standardized test. Conventional tests cannot reliably predict the way in which intelligence will be applied. Both traditional and modern psychometric theories face certain problems. First, it has not been proved that a truly general ability encompassing all mental abilities actually exists. Second, psychometric theories cannot precisely characterize all that goes on in the mind. Third, it is not clear whether the tests on which psychometric theories are based are equally appropriate in all cultures [17].

\section{CONCLUSION}

Intelligence has been a controversial subject throughout psychology's history. Intelligence is a quality that is unique to humans. Human intelligence is regarded as any information that can be gathered from human sources. It involves learning from previous experiences. This could be from education, work experiences, or a situation one goes through. Human intelligence increases by about three IQ points each decade due to improved health, education, and nutrition [18].

Although intelligence testing has emerged as a widely used tool, the debate continues today about whether accurate measurements of intelligence are even possible. More information about human intelligence can be found in the books in [19-38] and in the journal devoted to it: Intelligence.

\section{REFERENCES}

[1] K. Cherry, "Theories of intelligence in psychology," October 2019 ,

https://www.verywellmind.com/theories-ofintelligence-2795035

[2] "Human intelligence," Wikipedia, the free encyclopedia, https://en.wikipedia.org/wiki/Human_intelligence

[3] https://www.researchgate.net/figure/Threelevels-of-Human-IntelligenceSystem_fig1_305265106

[4] L. Gabora and A. Russon, (2011). "The evolution of human intelligence," In R. Sternberg and S. Kaufman (eds.), The Cambridge handbook of intelligence. Cambridge, UK: Cambridge University Press, chapter 17, 2011, pp. 328-350.

[5] "Intelligence quotient," Wikipedia, the free encyclopedia,https://en.wikipedia.org/wiki/Intellige nce_quotient

[6] A. Kuszewski, "You can increase your intelligence: 5 ways to maximize your cognitive potential," March 2011,

https://blogs.scientificamerican.com/guest-blog/youcan-increase-your-intelligence-5-ways-to-maximizeyour-cognitive-potential/

[7] "Artificial intelligence vs human intelligence," https://www.educba.com/artificial-intelligencevs-human-intelligence/

[8] M. A. Ali, "The human intelligence vs. artificial intelligence: Issues and challenges in computer assisted language learning," International Journal of English Linguistics; vol. 8, no. 5; 2018, pp. 259-271.

[9] K. S. Yeon, "Artificial intelligence vs Human," April 2017, http://eng.dt.co.kr/contents.html?article_no=20170

[10] K. Karachalios and J. Ito, "Human intelligence and autonomy in the era of 'extended intelligence,"' https://globalcxi.org/wpcontent/uploads/CXI_Essay.pdf

[11] E. Kamar, "Directions in hybrid intelligence: Complementing AI systems with human intelligence," Proceedings of the Twenty-Fifth International Joint Conference on Artificial Intelligence, 2016, pp. 40704073.

[12] J. St.Julien, "Changing conceptions of human intelligence and reasoning: Implications for the classroom," Australian Journal of Education, vol. 44, no.3, 2000, pp. 254- 271.

[13] R. D. Steele, "Human intelligence: All humans, all minds, all the time," Strategic Studies Institute, US Army War College, May 2010.

[14] T. Nunan et al., "Eliciting human intelligence: Police source handlers' perceptions and experiences of rapport during covert human intelligence sources (CHIS) interactions," Psychiatry, Psychology and Law, 2020.

[15] M. Li, W. Zhang, and X. Zhou, "Identification of genes involved in the evolution of human intelligence through combination of inter-species and interspecies genetic variations," PeerJ, vol. 8, April 2020.

[16] R. J. Cole, A. Bild, and E. Matheus, "Automated and human intelligence: Direct and indirect consequences," Intelligent Buildings International, vol. 4, no. 1, 2012, pp. 4-14.

[17] R. J. Sternberg, "Human intelligence," https://www.britannica.com/science/humanintelligence-psychology/Psychometric-theories

[18] "Human intelligence increases by three IQ points a decade,"https://zeenews.india.com/news/scitech/human-intelligence-increases-by-three-iqpoints-a-decade_1493434.html 
[19] R. J. Sternberg (ed.), Handbook of Human Intelligence. Cambridge, UK: Cambridge University Press, 2000.

[20] R. J. Sternberg (ed.), Human Intelligence: An Introduction. Cambridge, UK: Cambridge University Press, 2019.

[21] R. G. Steen, Human Intelligence and Medical Illness: Assessing the Flynn Effect. New York: Springer, 2009

[22] E. Hunt, Human Intelligence. Cambridge University Press, 2011.

[23] J. P. Guilford, The Nature of Human Intelligence. New York: McGraw-Hill, 1967.

[24] I. J. Deary, Looking Down on Human Intelligence: From Psychometrics to the Brain. Oxford University Press, 2000.

[25] C. Sagan, Dragons of Eden: Speculations On the Evolution of Human Intelligence. Ballantine Books, 1986.

[26] R. J. Sternberg and J. C. Kaufman (eds.), The Evolution of Intelligence. Psychology Press, 2013.

[27] N. Mackintosh, IQ and Human Intelligence. Oxford University Press, 2nd Edition, 2020.

[28] R. J. Sternberg and S. B. Kaufman(eds.), The Cambridge Handbook of Intelligence. Cambridge, UK: Cambridge University Press, 2011.

[29] R. J. Sternberg and P. B. Baltes, International Handbook of Intelligence. Cambridge, UK: Cambridge University Press, 2004.

[30] H. Moravec, Mind Children: The Future of Robot and Human Intelligence. Cambridge: Harvard University Press, 1988.

[31] J. Bailey, After Thought: The Computer Challenge to Human Intelligence. New York: HarperCollins Publishers, 1996.
[32] H. J Butcher, Human Intelligence: Its Nature and Assessment. Methuen and Co., 1968.

[33] R. Sternberg, Beyond IQ: A Triarchic Theory of Human Intelligence. Cambridge University Press, 1984.

[34] R. Billingsley, Covert Human Intelligence Sources: The 'Unlovely' Face of Police Work. Waterside Press, 2009.

[35] The Biology of Human Intelligence: Proceedings of the Twentieth Annual Symposium of the Eugenics Society. London, 1983.

[36] R. J. Sternberg (ed.), The Nature of Human Intelligence. Cambridge University Press, 2018.

[37]_D. Ram, Dynamics of Human Life: A book on Human Intelligence. Amazon.com Services, 2018.

\section{AUTHORS}

Matthew N.O. Sadiku is a professor emeritus in the Department of Electrical and Computer Engineering at Prairie View A\&M University, Prairie View, Texas. He is the author of several books and papers. His areas of research interests include computational electromagnetics and computer networks. He is a fellow of IEEE.

Tolulope J. Ashaolu works at Southwest University. He is the author of several papers and books. His research interests include functional foods and food microbiology. Sarhan M. Musa is a professor in the Department of Electrical and Computer Engineering at Prairie View A\&M University, Prairie View, Texas. He has been the director of Prairie View Networking Academy, Texas, since 2004. He is an LTD Sprint and Boeing Welliver Fellow. His areas of research interests include computational electromagnetics and computer networks.

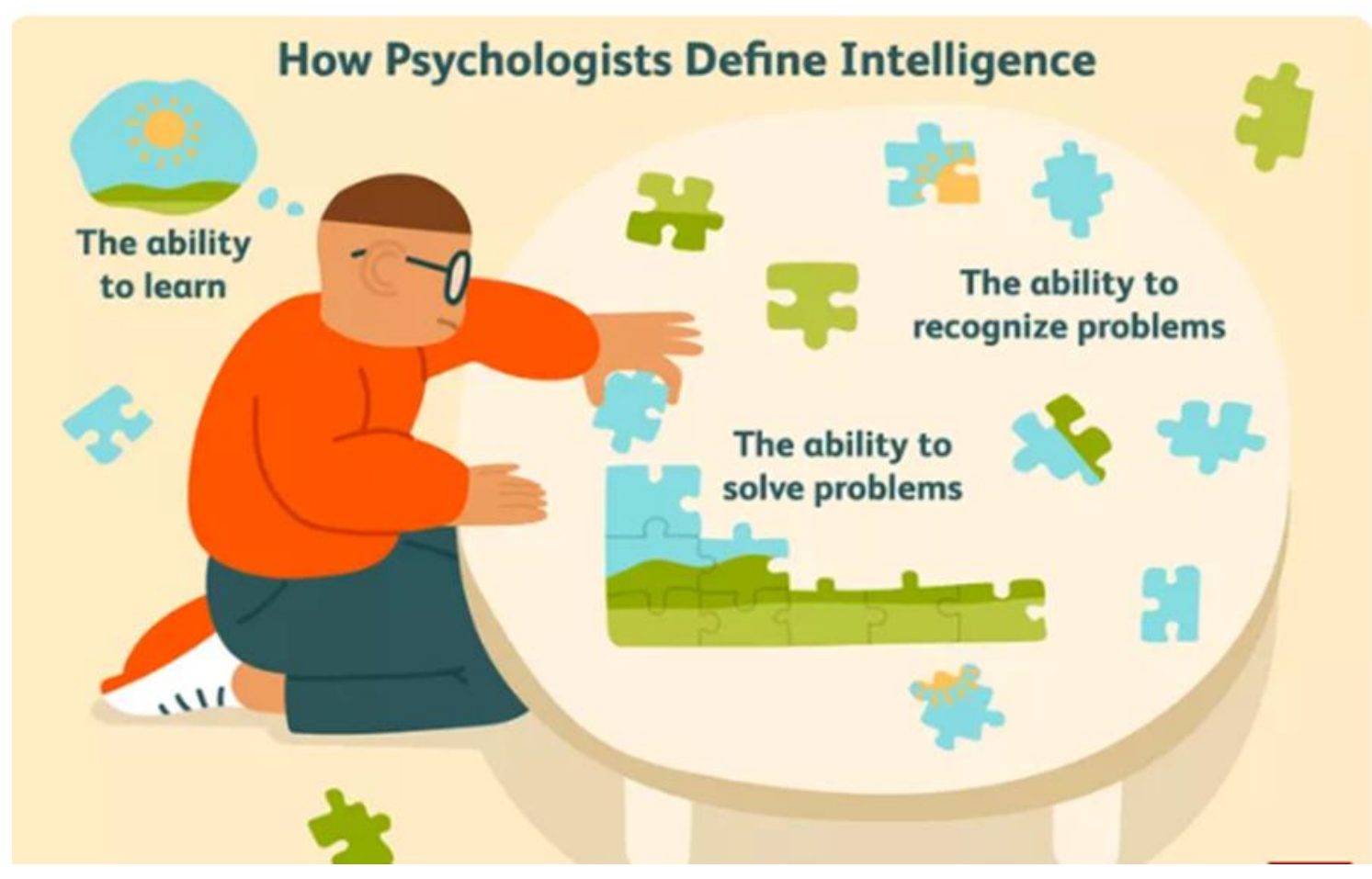

FIGURE 1: HOW PSYCHOLOGIES DEFINE OINTELLIGENCE [1]. 


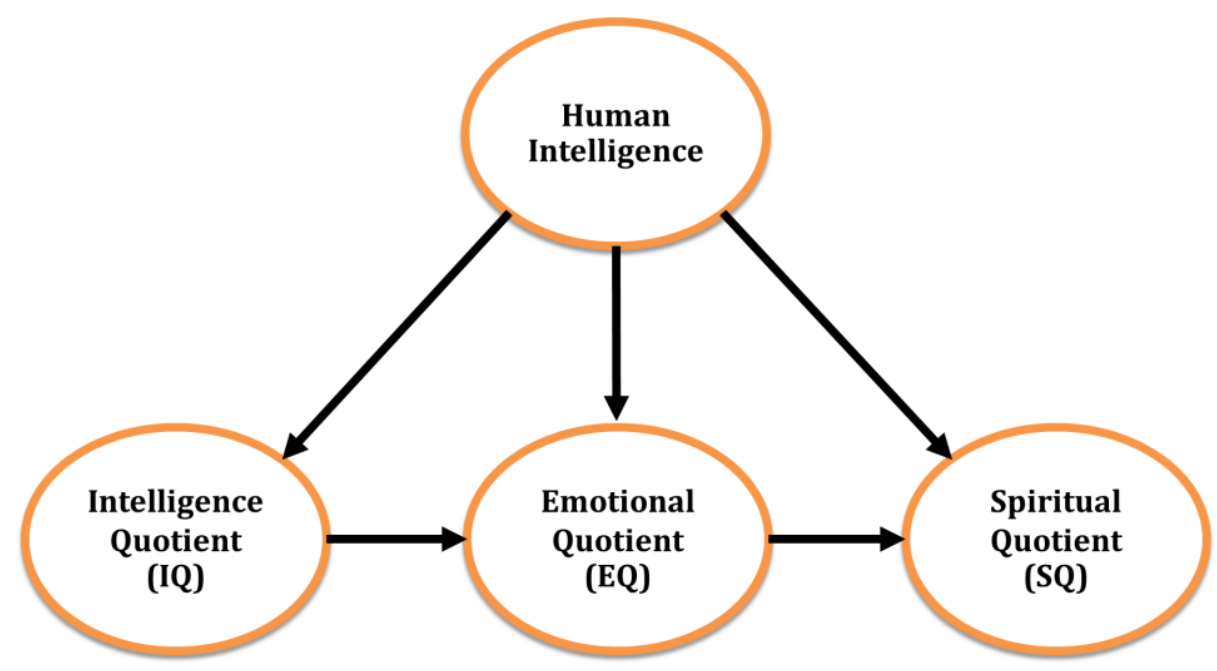

FIGURE 2: THREE COMPONENT OF HUMAN INTELLIGENCE [3].

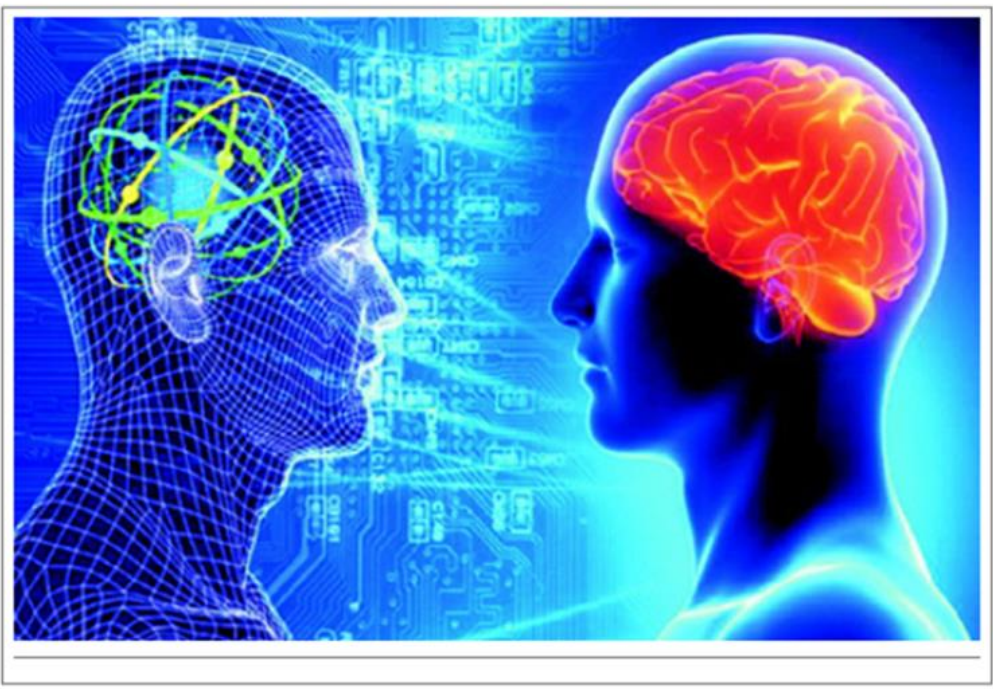

FIGURE 3: ARTIFICIAL INTELLIGENCE VERSUS HUMAN INTELLIGENCE [9].

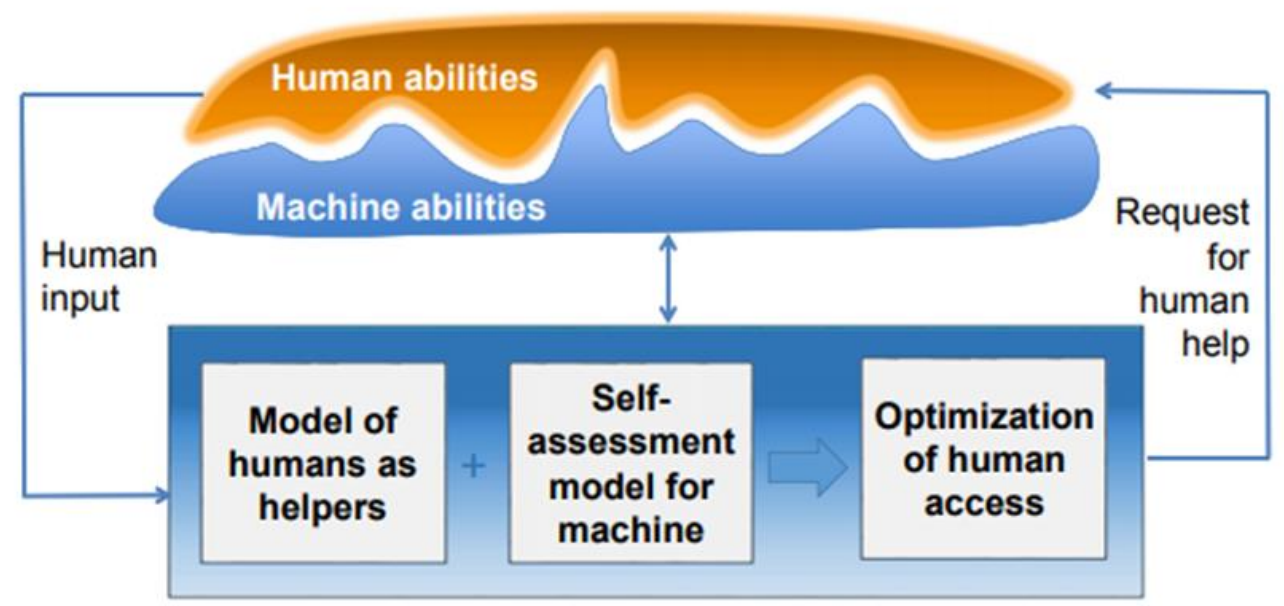

FIGURE 4: REASONING CAPABILITIES FOR HYBRID INTELLIGENCE [11]. 